\title{
Necessary Auxiliary Background for Efficient Use of an Existing Computer Program of Non-parametric Fitting of Nonlinear Equations
}

\author{
Emmanuel M. Papamichael" and Leonidas G. Theodorou \\ Sector of Organic Chemistry and Biochemistry; Laboratory of Enzymology; Department Chemistry; University of
} Ioannina 45110, Ioannina - GREECE

\begin{abstract}
This work constituted a significant contribution for more efficient use of a valuable computer program of nonparametric fitting of nonlinear multiparametric equations to experimental data. However, prerequisite in this context was the transformation of nonlinear multiparametric equations into linear hyperplane forms before their incorporation within the computer program; this latter was decisive and a matter of proper programming practice. Herein, a series of widely used equations useful in different fields of chemical processes, in biochemistry and/or in biotechnology, along with their suitable transformations as well as the appropriate programming support are being reported.
\end{abstract}

Key Words: Non-parametric methods, computer program, fitting of nonlinear equations

\section{INTRODUCTION}

A non-parametric method of fitting nonlinear multiparametric equations to experimental data, along with a suitable computer program, has been presented in details and analyzed statistically previously. However, the transformation of nonlinear multiparametric equations into linear hyperplane forms, before their incorporation within the computer program is a prerequisite (Papamichael et al., 2000; Eisenthal and CornishBowden, 1974). This latter is a matter of proper programming practice, which could obstruct the use of the non-parametric fitting program, and it is not uncommon in cases where the monitored response of a chemical, biochemical and/or biotechnological process is described by a more or less awkward equation (Theodorou et al., 2001,
2007). Herein, we report a series of widely used equations in the fields of chemistry, biochemistry and biotechnology, along with their suitable transformations and the appropriate programming support.

\section{Equations}

All nonlinear multiparametric equations appeared in Table 1 are frequently and commonly encountered in chemical, biochemical and/or biotechnological processes. As a matter of fact, a larger number of equations could be cited in this report; however we collected those which were considered as relatively more important due to their usefulness. On the other hand the transformations given below could be applied to other equations having similar forms to those cited herein.

\footnotetext{
* Author for correspondence: epapamic@cc.uoi.gr
} 
Table 1

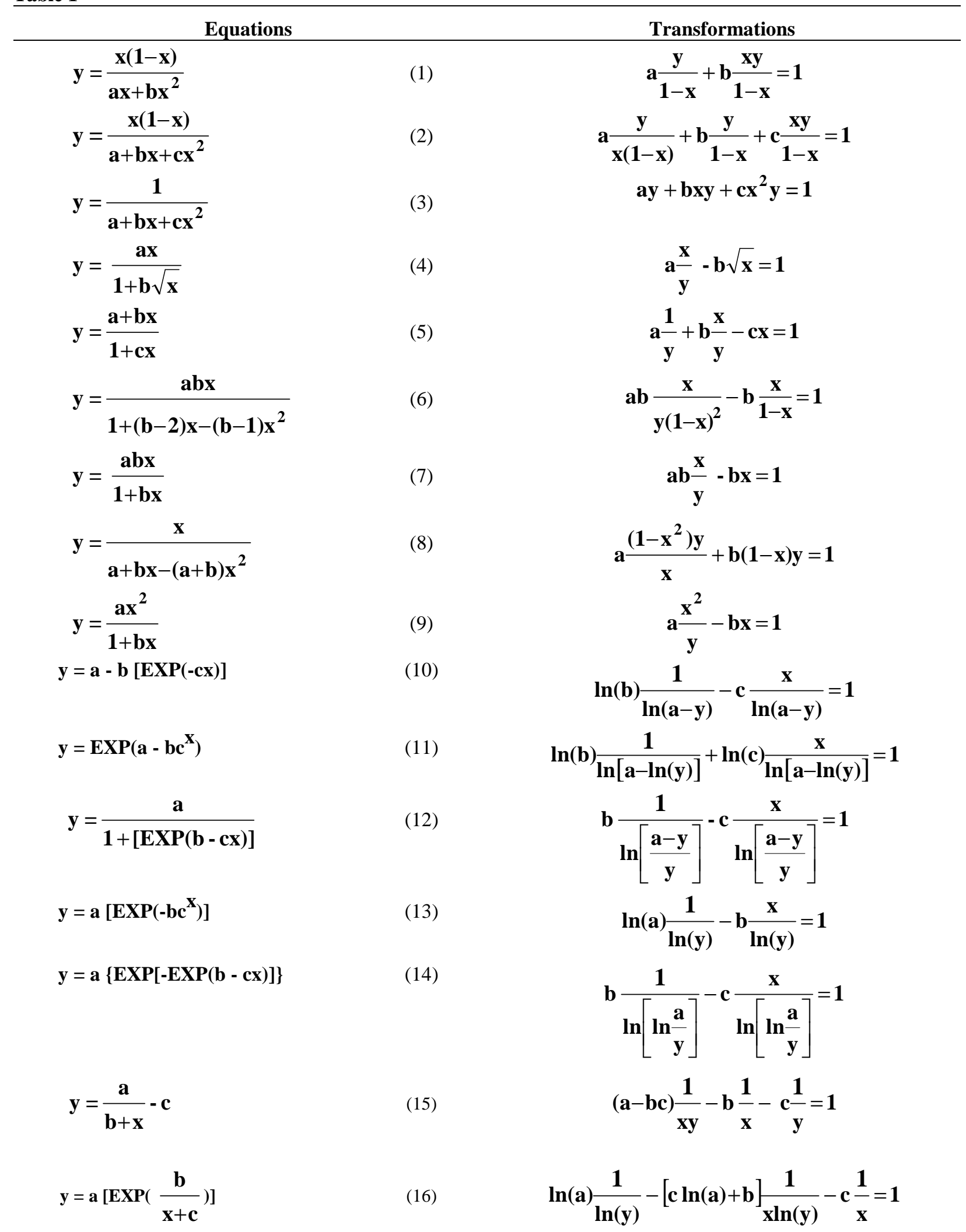




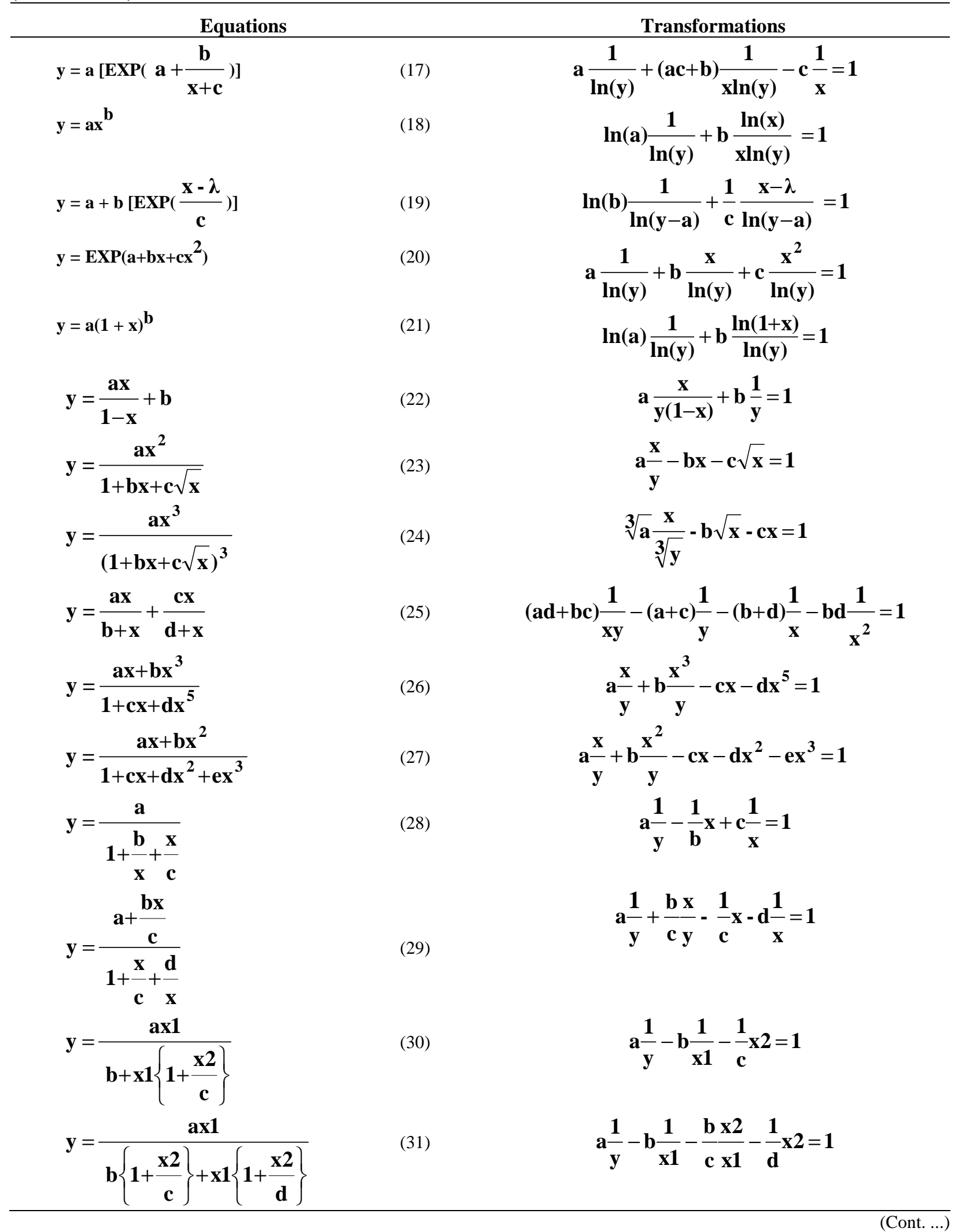




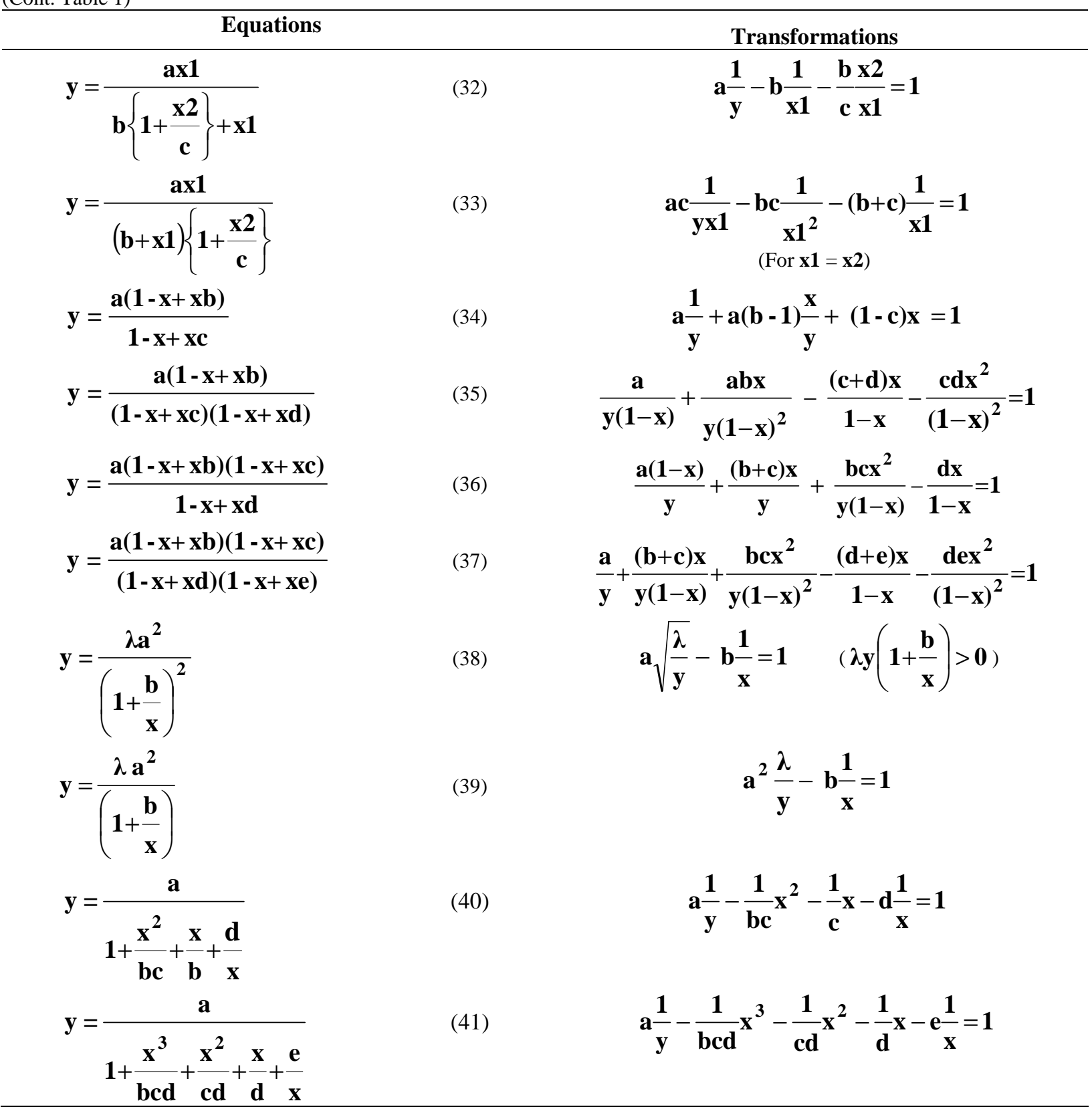

Equations (1) to (24) are referred to chemical and/or biotechnological procedures, as the chlorination of di-chloro-hydrocarbons to tri- and tetra- analogues, and/or to yield-density, sigmoidgrowth and logistic models of the development of certain organisms (Kafarov, 1976; Iglesias and Chirife, 1981; Ratkowsky, 1983; Pilling and Seakins, 1997; Papamichael et al., 2000). However, equations (25) to (33) are frequently encountered in biochemical reactions (DoubleMichaelis, non-Michaelis, reversible inhibition and/or activation and uptake kinetics); equations
(34) to (39) are important in proton inventories and/or in burst kinetics (Papamichael and Lymperopoulos 1988; Theodorou et al., 2001, 2007). Similarly, equations (40) and (41), including equation (29), are useful in fitting experimental data from $\mathrm{pH}$-profiles, and are valuable in treating biotechnological data Theodorou et al., 2007).

\section{Transformations}

The transformations which are illustrated herein are based on the fundamental requirements of $\mathbf{y \neq 0}$ 
and/or $\mathbf{x} \neq \mathbf{0}$, i.e. both the dependent and the independent variables never take a zero-value, as this is valid under the experimental conditions in chemistry, biochemistry, biotechnology etc. In the same way, we should point out how simply and easily were performed all transformations depicted in Table 1. Some additional difficulties were faced in cases of equations (10), (11), (12), (14) and (19), where a parameter, namely $\boldsymbol{a}$, was incorporated into the dependent variable during their transformation for simplicity purposes. In following are given, as examples, the transformations of equation (1), as well as of some other awkward equations.

Equation (1) can be transformed to the form $\mathbf{a x y}+$ $\mathbf{b x}^{2} \mathbf{y}=\mathbf{x}(\mathbf{1 - x}) \quad($ as $\mathbf{y \neq 0}$ and/or $\mathbf{x} \neq \mathbf{0})$; then, by dividing both members of its new form by $\mathbf{x}(\mathbf{1}-\mathbf{x})$, and performing all necessary simplifications the suitable form $\mathbf{a} \frac{\mathbf{y}}{\mathbf{1}-\mathbf{x}}+\mathbf{b} \frac{\mathbf{x y}}{\mathbf{1 - x}}=\mathbf{1}$ is the result.

Alternatively, in equation (10) the first step is the separation of its variables, where a new equation a - $\mathbf{y}=\mathbf{b}[\mathbf{E X P}(-\mathbf{c x})]$ is formed, whose both logarithmic forms $\ln (\mathbf{a}-\mathbf{y})=\mathbf{- c x}+\ln (\mathbf{b})$ and $\ln (\mathbf{b})$ - $\mathbf{c x}=\ln (\mathbf{a}-\mathbf{y})$ are valid. The transformation of equation (10) is easily obtained by dividing both members of the latter equation by $\ln (\mathbf{a}-\mathbf{y})$.

In equation (11) the first step is to form the equality $\ln (\mathbf{y})=\mathbf{a}-\mathbf{b} \mathbf{c}^{\mathbf{x}}$; the separation of the variables comes next, i.e. $\mathbf{a}-\ln (\mathbf{y})=\mathbf{b c} \mathbf{x}$, and equation $\ln [\mathbf{a}-\ln (\mathbf{y})]=\ln (\mathbf{b})+\mathbf{x} \ln (\mathbf{c})$ is obtained which is easily transformed to its suitable form $\ln (\mathbf{b}) \frac{1}{\ln [\mathbf{a}-\ln (\mathbf{y})]}+\ln (\mathbf{c}) \frac{\mathbf{x}}{\ln [\mathbf{a}-\ln (\mathbf{y})]}=1$. By performing very similar rearrangements one may transform appropriately equations (12) and (19); however, a last example should be given, that of equation (14).

In equation (14), both its members were divided by a to obtain $\frac{\mathbf{y}}{\mathbf{a}}=\mathbf{E X P}[-\operatorname{EXP}(\mathbf{b}-\mathbf{c x})]$ and then $\ln \left[\frac{\mathbf{y}}{\mathbf{a}}\right]=-\mathbf{E X P}(\mathbf{b}-\mathbf{c x})$, which was rearranged to the form $\ln \left[\frac{\mathbf{a}}{\mathbf{y}}\right]=\mathbf{E X P}(\mathbf{b}-\mathbf{c x})$. If and only if $\frac{\mathbf{y}}{\mathbf{a}}>\mathbf{1}$, then the latter equation was transformed to $\ln \left[\ln \frac{\mathbf{a}}{\mathbf{y}}\right]=\mathbf{b}-\mathbf{c x}$, by taking the logarithms, and then taken on its final form $\mathbf{b} \frac{\mathbf{1}}{\ln \left[\ln \frac{\mathrm{a}}{\mathrm{y}}\right]}-\mathbf{c} \frac{\mathbf{x}}{\ln \left[\ln \frac{\mathrm{a}}{\mathrm{y}}\right]}=\mathbf{1}$, as before.

Hence, in cases of equations (10), (11), (12), (14) and (19) initial guessing values of parameter $\boldsymbol{a}$ must be obtained easily and incorporated within transformations for further use by the computer program; this holds true and it needs only a simple inspection of the experimental points in a scatter graph (Cornish-Bowden, 1995). Useful examples are depicted in Figures 1 and 2, where estimates of parameter $\boldsymbol{a}$ can be obtained: (a) as the maximum value of the independent variable $\boldsymbol{y}$ when $\boldsymbol{x} \rightarrow \infty$ (asymptote parallel to abscissas i.e. the Y-axis), in cases of equations (10), (12) and (14) (Fig. 1 and 2 ), (b) by subtracting the value of numeric constant $\lambda$ from that of the intersection of the experimental curve on the ordinate axis (Fig. 1) in case of equation (19), and (c) as the natural logarithm (ln) of the maximum value of the independent variable $\boldsymbol{y}$ when $\boldsymbol{x} \rightarrow \infty$ in case of equation (11) (Fig. 2).

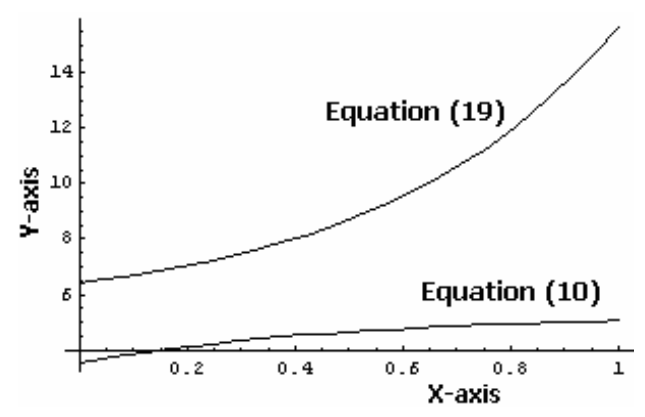

Figure 1 - Equations (10) and (19) were drawn as: $\mathbf{y}=\mathbf{5 . 3}$ - $\mathbf{1 . 7 5}[\mathbf{E X P}(-\mathbf{1 . 9 5 x})]$, and $\mathbf{y}=\mathbf{5 . 3}+$ $1.75\left[\operatorname{EXP}\left(\frac{\mathrm{x}-0.2}{0.45}\right)\right]$, respectively. 


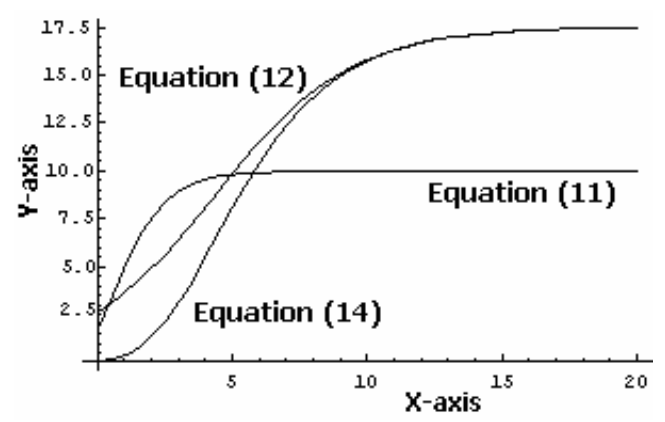

Figure 2 - Equations (11), (12) and (14) were drawn as: $\mathbf{y}=\mathbf{E X P}\left(\mathbf{2 . 3}-\mathbf{1 . 7 5} * \mathbf{0 . 4}{ }^{\mathbf{X}}\right.$ ), $\mathbf{y}=\frac{17.5}{1+[\operatorname{EXP}(1.75-0.4 x)]}$, and $\mathbf{y}=17.5\{\operatorname{EXP}[-\operatorname{EXP}(1.75-0.4 x)]\}$, respectively.

In this way, transformations were provided for all the above mentioned nonlinear multiparametric equations into their linear hyperplane forms. In the above equations and their transformations, $\lambda$ is only a numeric constant. Additionally, care should be taken in transformations when the natural logarithm (ln) of an expression is appeared; these expressions should take on values only greater than zero.

\section{RESULTS AND DISCUSSION}

What could be deduced from the preceding section was that which has been mentioned about the prerequisites of using the previously described computer program (Papamichael et al., 2000). Therefore, in this work, a series of nonlinear multiparametric equations, useful in different fields of sciences and technology, were properly transformed into linear hyperplane forms, capable for incorporation within this program. Then, to accomplish successfully the non-parametric fitting of a nonlinear multiparametric equation, after its suitable transformation, one has only to follow the build-in instructions mentioned in the program listing.

Let us take equation (1) as an example along with its linear transformation, i.e. equation $\mathbf{a} \frac{\mathbf{y}}{\mathbf{1}-\mathbf{x}}+\mathbf{b} \frac{\mathbf{x y}}{\mathbf{1}-\mathbf{x}}=\mathbf{1}$. Then, in addition to the details given previously (Papamichael et al., 2000), the program user should form carefully a system of simultaneous equations found within the multiple FOR-NEXT loops by following the appropriate syntax. In the example, the program user should type two lines as indicated below, and should not confuse e with the base of natural logarithms, in e!(i,j) statements:

First line: e!(1,1)=y!(op)/(1-x!(op)):

$\mathbf{e} !(1,2)=x !(o p) * y !(o p) /(1-x !(o q))): e !(1,3)=1$

Second line: $\quad \mathbf{e} !(2,1)=y !(o q) /(1-x !(o q))$ : $\mathrm{e} !(2,2)=x !(o q) * y !(o q) /(1-x !(o q)): e !(2,3)=1$

It should be emphasized that the MichaelisMenten equation $\mathbf{y}=\frac{\mathbf{a x}}{\mathbf{b}+\mathbf{x}}$, as well as equation (23) of this report, have been presented and accordingly analyzed previously (Eisenthal and Cornish-Bowden, 1974; Papamichael et al., 2000)

\section{REMARKS}

The program listing has been developed under ZBASIC compiler for Macintosh, and it is given below. However, there is a version of the ZBASIC compiler for PC-compatible computers, and authors could help in a future appropriate transforming of the program listing.

Likewise, the principles of transformation of nonlinear multiparametric equations into linear hyperplane forms, as well as a complete statistical analysis and description and function of the computer program have been already described in details previously (Papamichael et al., 2000). 


\section{The Program Listing and an Example}

REM Non-Parametric Curve Fitting. Configure ZBASIC for Integer Variables.

REM Clear, and Clear (binco+2)*(number of digits of ndp +1)*npr (see a $\$=$. . below).

CLEAR: CLEAR 12100

ndp=14:npr=4:binco $=1001:$ cntr $=0$

REM DIM's: x(ndp), y(ndp), r(ndp), e(npr+1,ndp+1), g(npr+1), pm(npr,binco), vl(npr,6), dx(npr)

DIM x!(14),y!(14),r!(14),e!(5,15),g!(5),pm!(4,1001),vl!(4,6), dx(4)

INDEX\$(0)=""

FOR $\mathrm{j}=1$ TO ndp

READ $x !(j), y !(j)$

NEXT j

CLS: PRINT TIME\$: REM Time\$ function Optional

FOR op $=1$ TO ndp

FOR oq=1 TO ndp

IF (oq=op) THEN "NextA"

FOR or $=1$ TO ndp

IF ((or=oq) OR (or=op)) THEN "NextB"

FOR ow $=1$ TO ndp

IF ((ow=or) OR (ow=oq) OR (ow=op)) THEN "NextC"

$\mathrm{dx}(1)=\mathrm{op}$

$\mathrm{dx}(2)=\mathrm{oq}$

$\mathrm{dx}(3)=$ or

$\mathrm{dx}(4)=\mathrm{ow}$

GOSUB "Sortindices"

$\mathrm{a} \$=\mathbf{S T R} \$(\mathrm{dx}(1))+\mathbf{S T R} \$(\mathrm{dx}(2))+\mathbf{S T R} \mathbf{T}(\mathrm{dx}(3))+\mathbf{S T R} \mathbf{T}(\mathrm{dx}(4))$

REM a $\$$-> (number of digits of ndp +1$) *$ npr

IF INDEXF $(a \$)=-1$ THEN INDEX $\$($ cntr+1)=a $\$$ ELSE "NextC" cntr $=$ cntr +1

$\mathrm{e} !(1,1)=x !(\text { op })^{\wedge} 2 / y !(o p): \quad e !(1,2)=-x !(o p)^{\wedge} 2: e !(1,3)=-x !(o p): \quad e !(1,4)=-S Q R(x !(o p)): \quad e !(1,5)=1$ $\mathrm{e} !(2,1)=x !(\mathrm{oq})^{\wedge} 2 / y !(\mathrm{oq}): \mathrm{e} !(2,2)=-\mathrm{x} !(\mathrm{oq})^{\wedge} 2: \mathrm{e} !(2,3)=-\mathrm{x} !(\mathrm{oq}): \mathrm{e} !(2,4)=-\mathbf{S Q R}(\mathrm{x} !(\mathrm{oq})): \mathrm{e} !(2,5)=1$ $\mathrm{e} !(3,1)=\mathrm{x} !(\text { or })^{\wedge} 2 / \mathrm{y} !($ or $): \quad \mathrm{e} !(3,2)=-\mathrm{x} !(\text { or })^{\wedge} 2: \mathrm{e} !(3,3)=-\mathrm{x} !($ or $): \quad \mathrm{e} !(3,4)=-\mathbf{S Q R}(\mathrm{x} !($ or $)): \quad \mathrm{e} !(3,5)=1$ $\mathrm{e} !(4,1)=\mathrm{x} !(\mathrm{ow})^{\wedge} 2 / \mathrm{y} !(\mathrm{ow}): \mathrm{e} !(4,2)=-\mathrm{x} !(\mathrm{ow})^{\wedge} 2: \mathrm{e} !(4,3)=-\mathrm{x} !(\mathrm{ow}): \mathrm{e} !(4,4)=-\mathbf{S Q R}(\mathrm{x} !(\mathrm{ow})): \mathrm{e} !(4,5)=1$

GOSUB "Cholesky"

FOR os=1 TO npr

REM Assignment for future sorting of the parameters estimates.

$\mathrm{pm} !(\mathrm{os}, \mathrm{cntr})=\mathrm{g} !(\mathrm{os})$

NEXT os

"NextC"

NEXT ow

"NextB"

NEXT or

"NextA"

\section{NEXT oq,op}

REM Procedure to Sort Parameter Estimates; Shell-Metzner Type.

FOR $\mathrm{jj}=1$ TO npr: REM For All parameter series.

meo=binco

"CycA"

meo $=\mathbf{I N T}(\mathrm{meo} / 2)$

IF meo=0 THEN "CycE"

jaj=1:kaj=binco-meo

"CycB"

iaj=jaj

"CycC"

laj=iaj+meo

IF pm!(jj,iaj)<=pm!(jj,laj) THEN "CycD" ELSE SWAP pm!(jj,iaj),pm!(jj,laj)

iaj=iaj-meo

IF iaj<1 THEN "CycD" 


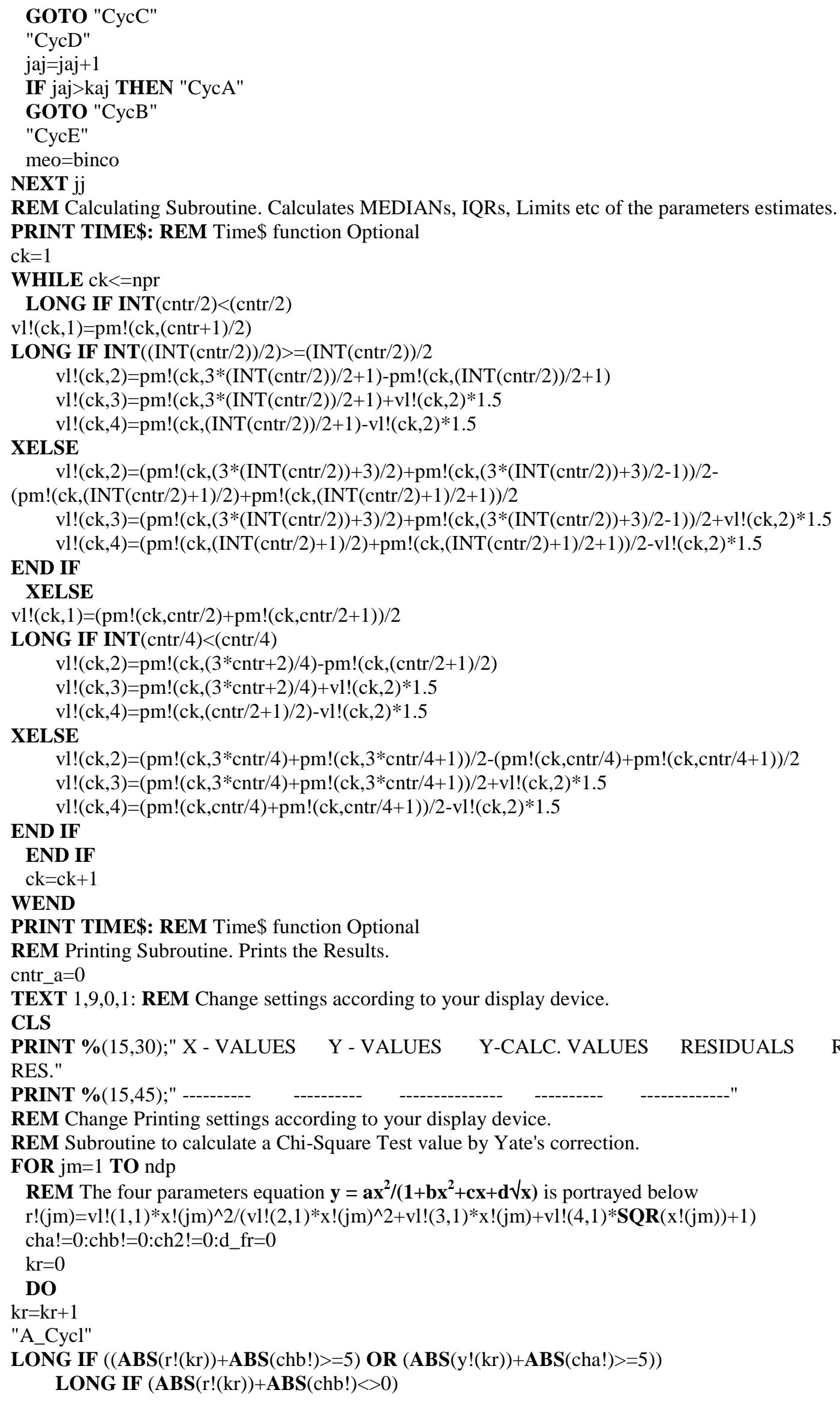

PRINT \%(15,45);"

FOR $\mathrm{jm}=1$ TO $\mathrm{ndp}$

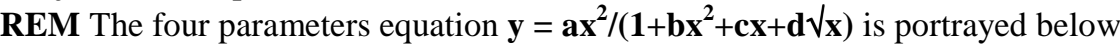

$\mathrm{r} !(\mathrm{jm})=\mathrm{vl} !(1,1)^{*} \mathrm{x} !(\mathrm{jm})^{\wedge} 2 /\left(\mathrm{vl} !(2,1)^{*} \mathrm{x} !(\mathrm{jm})^{\wedge} 2+\mathrm{vl} !(3,1)^{*} \mathrm{x} !(\mathrm{jm})+\mathrm{vl} !(4,1)^{*} \mathbf{S Q R}(\mathrm{x} !(\mathrm{jm}))+1\right)$

cha $!=0: \operatorname{chb} !=0: \operatorname{ch} 2 !=0: \mathrm{d}$ fr $=0$

$\mathrm{kr}=0$

$\mathrm{kr}=\mathrm{kr}+$

"A Cycl"

LONG IF (ABS $(\mathrm{r} !(\mathrm{kr}))+\mathbf{A B S}(\mathrm{chb} !)<>0)$ 


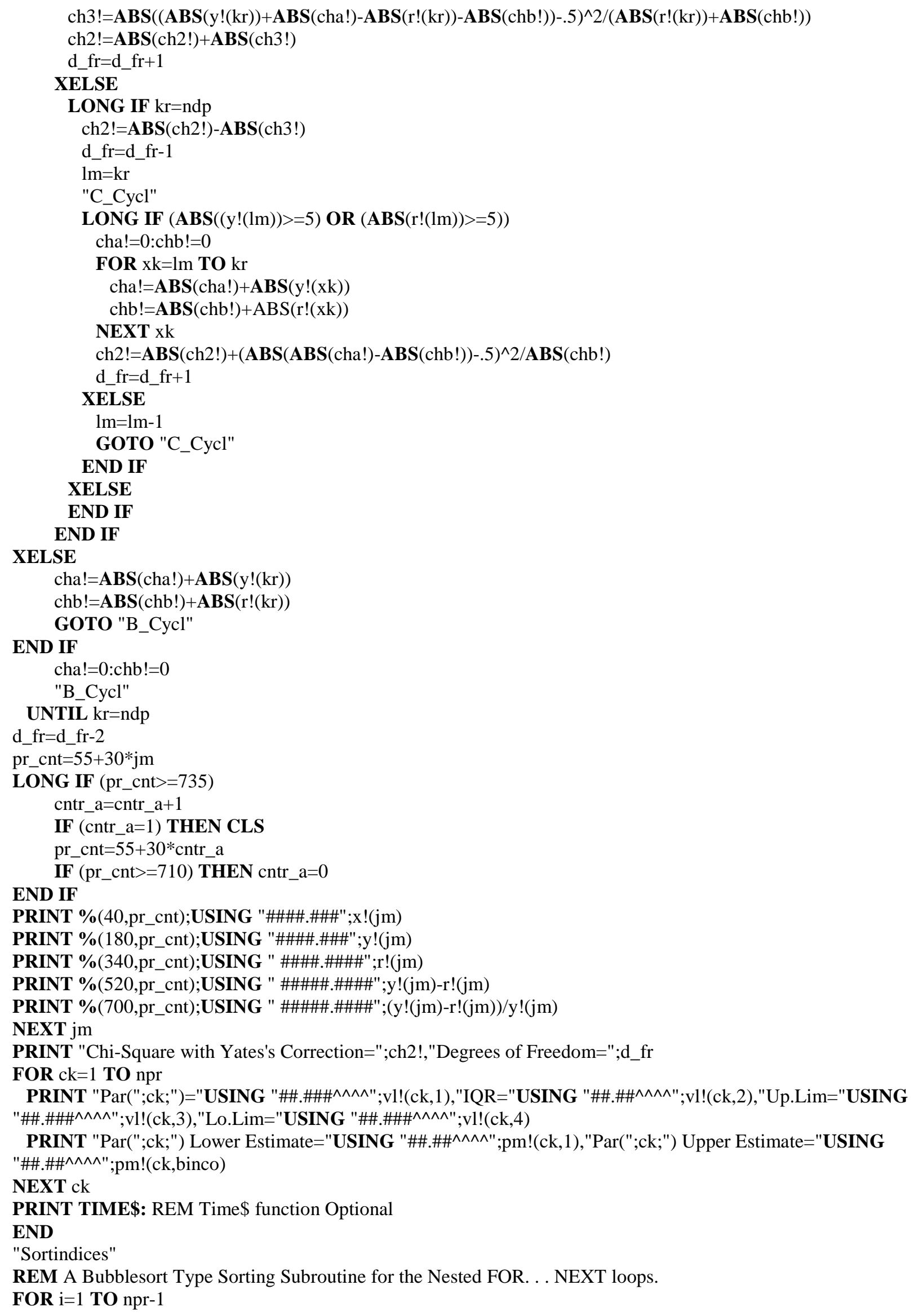




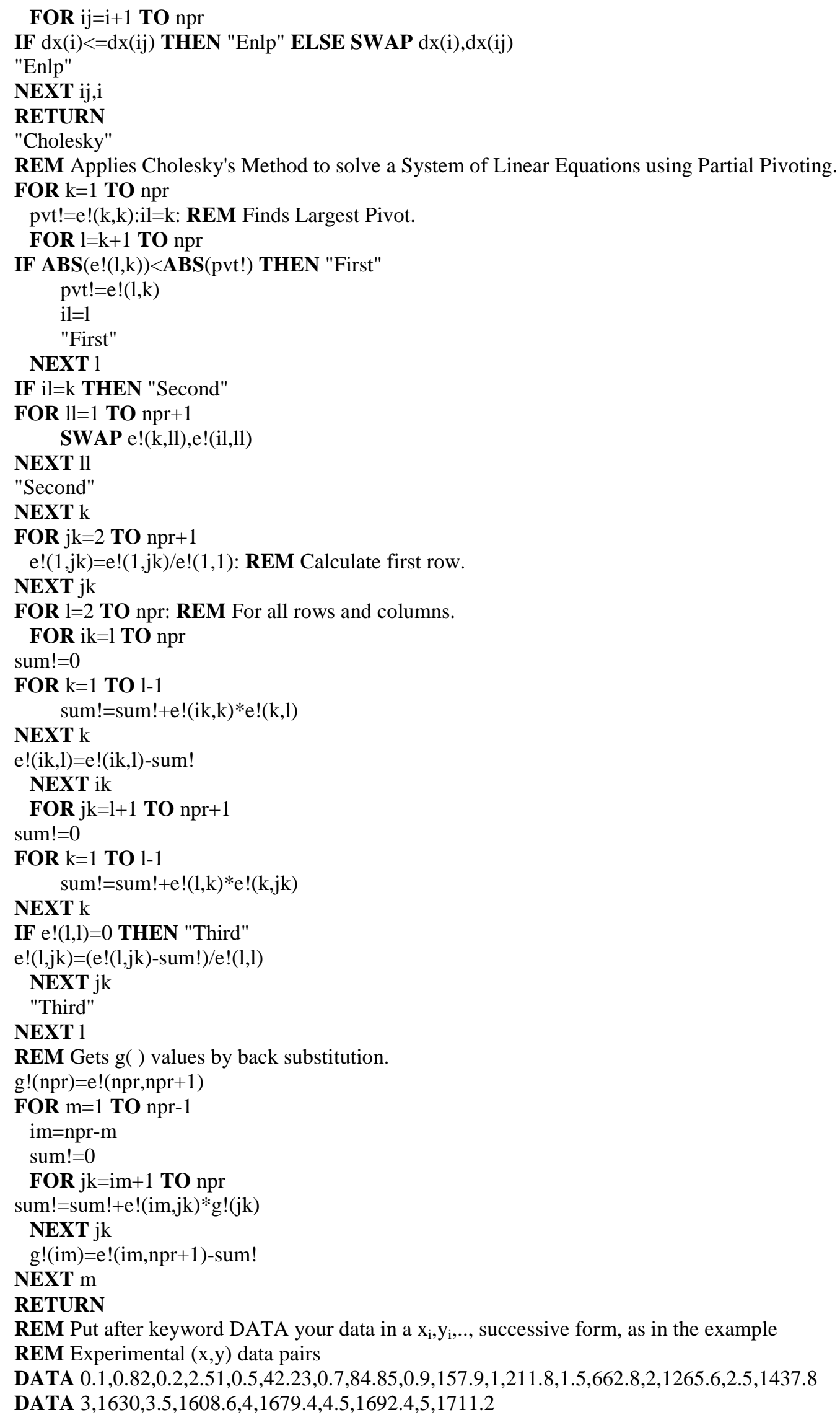


REM If more than one independent variables are provided by your equation, then put your data in a $\mathrm{x} 1_{\mathrm{i}}$, $\mathrm{x} 2{ }_{\mathrm{i}}, \ldots$ etc.. $\mathrm{y}_{\mathrm{i}}, .$. , successive form, and PROVIDE as more as you need $\mathrm{x} 1 !(), \mathrm{x} 2 !() \ldots$ etc DIM in the DIM-statements line, as well as in the READ-statement line.

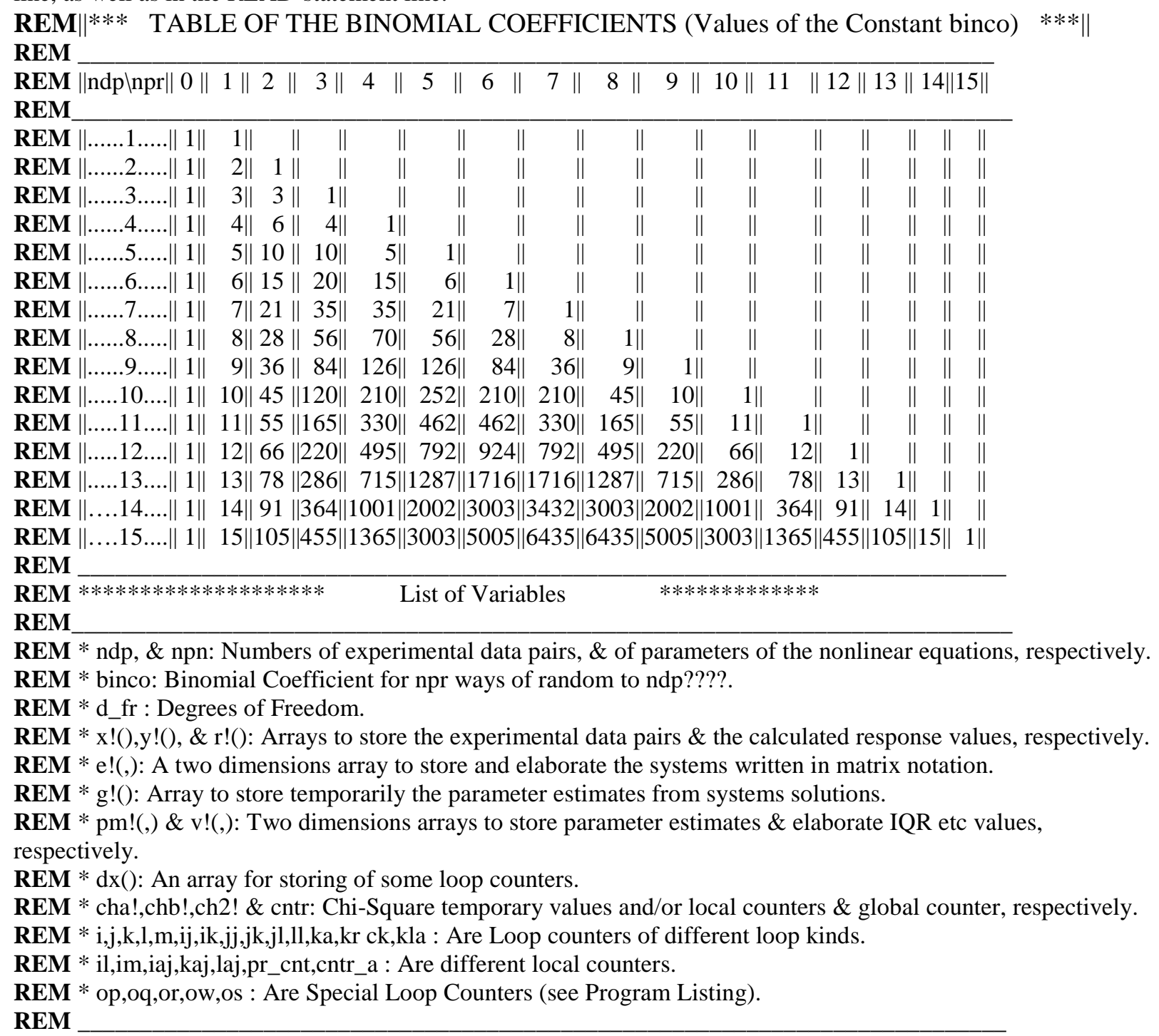

\section{RESUMO}

Este trabalho constitui uma contribuição significativa para um uso mais eficiente de um programa de computador valioso do encaixe nãoparamétrico de equações multiparamétricas não lineares aos dados experimentais. Entretanto, o pré-requisito neste contexto é a transformação de equações multiparamétricas não lineares em formulários lineares do hyperplano antes de sua incorporação dentro do programa de computador; este último é decisive e uma matéria da prática de programação apropriada. Nisto, nós relatamos uma série das equações extensamente usadas úteis em campos diferentes de processos químicos, em bioquímia e/ou em biotechnologia, junto com suas transformações apropriadas além a sustentação de programação apropriada.

\section{REFERENCES}

Cornish-Bowden, A. (1995), Analysis of Enzyme Kinetic Data. Oxford University Press, Oxford, pp.39.

Eisenthal, R., and Cornish-Bowden, A. (1974), Statistical considerations in the estimation of enzyme kinetic parameters by the direct linear plot and other methods. Biochem. J., 139, 721-730. 
Iglesias, H.A., and Chirife, J. (1981), An Equation for Fitting Uncommon Water Sorption Isotherms in Food. Lebens. Wiss. Technol., 14, 105-106.

Kafarov, V. (1976), Cybernetic Methods in Chemistry \& Chemical Engineering, MIR Publishers, Moscow, pp. 374-379 (Translated in English).

Papamichael, E.M., and Lymperopoulos, K. (1998), Elastase and Cathepsin-G from Human PMN Activated by PAF: Relation between their Kinetic Behavior and Pathophysiological Role. In Advances in Biotechnology, ed. A. Pandey. Educational Publishers and Distributors, New Delhi, pp.221-234.

Papamichael, E.M., Evmiridis, N.P., and Potosis, C. (2000), Non-parametric Fitting of Nonlinear Equations to Experimental Data without Use of Initial Guessing: A Basic Computer Program. Brazilian Archives of Biology and Technology, 43, 1-9.

Pilling, M.J., and Seakins, P.W. (1997), Reaction Kinetics. Oxford University Press, Oxford, pp.190200.
Ratkowsky, D.A. (1983), Nonlinear Regression Modeling: A Unified Practical Approach. Marcel Dekker Inc, New York - Basel, pp. 49-134.

Theodorou, L.G., Lymperopoulos, K., Bieth, J.G., and Papamichael, E.M. (2001), Insight into the Catalysis of Hydrolysis of Four Newly Synthesized Substrates by Papain: A Proton Inventory Study. BiochemistryUS, 40, 3996-4004.

Theodorou, L.G., Perysinakis, A., Valasaki, K., Drainas, C., and Papamichael, E.M. (2007), Proton inventories constitute reliable tools in investigating enzymatic mechanisms: application on a novel thermo-stable extracellular protease from a haloalkaliphilic Bacillus sp. J. Biochem. (Tokyo), 142, 293-300.
Received: March 03, 2008; Revised: September 03, 2008; Accepted: December 11, 2008. 